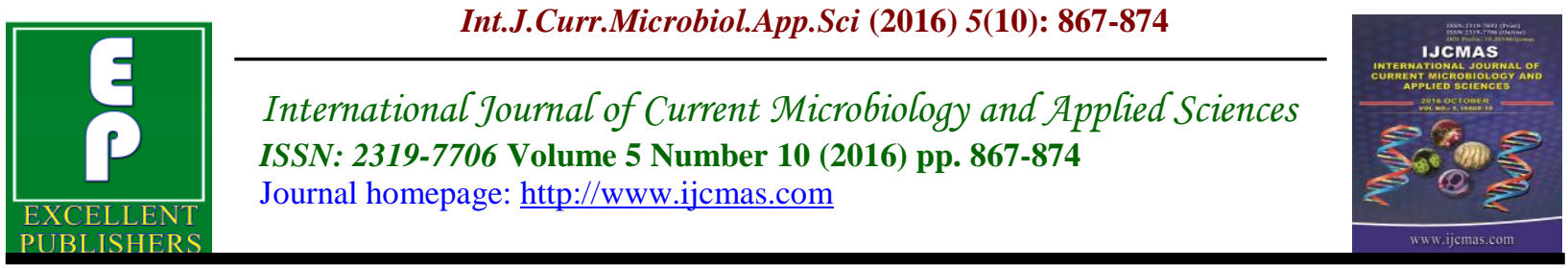

Original Research Article

http://dx.doi.org/10.20546/ijcmas.2016.510.095

\title{
C - Reactive Protein Test and Buffy Coat Smear Examination in Early Diagnosis of Neonatal Septicemia
}

\author{
Archana P. Nikam* and Pramod R. Bhise \\ Department of Microbiology, Dr. P.D.M. Medical College, Amravati, \\ Maharashtra State, India \\ *Corresponding author
}

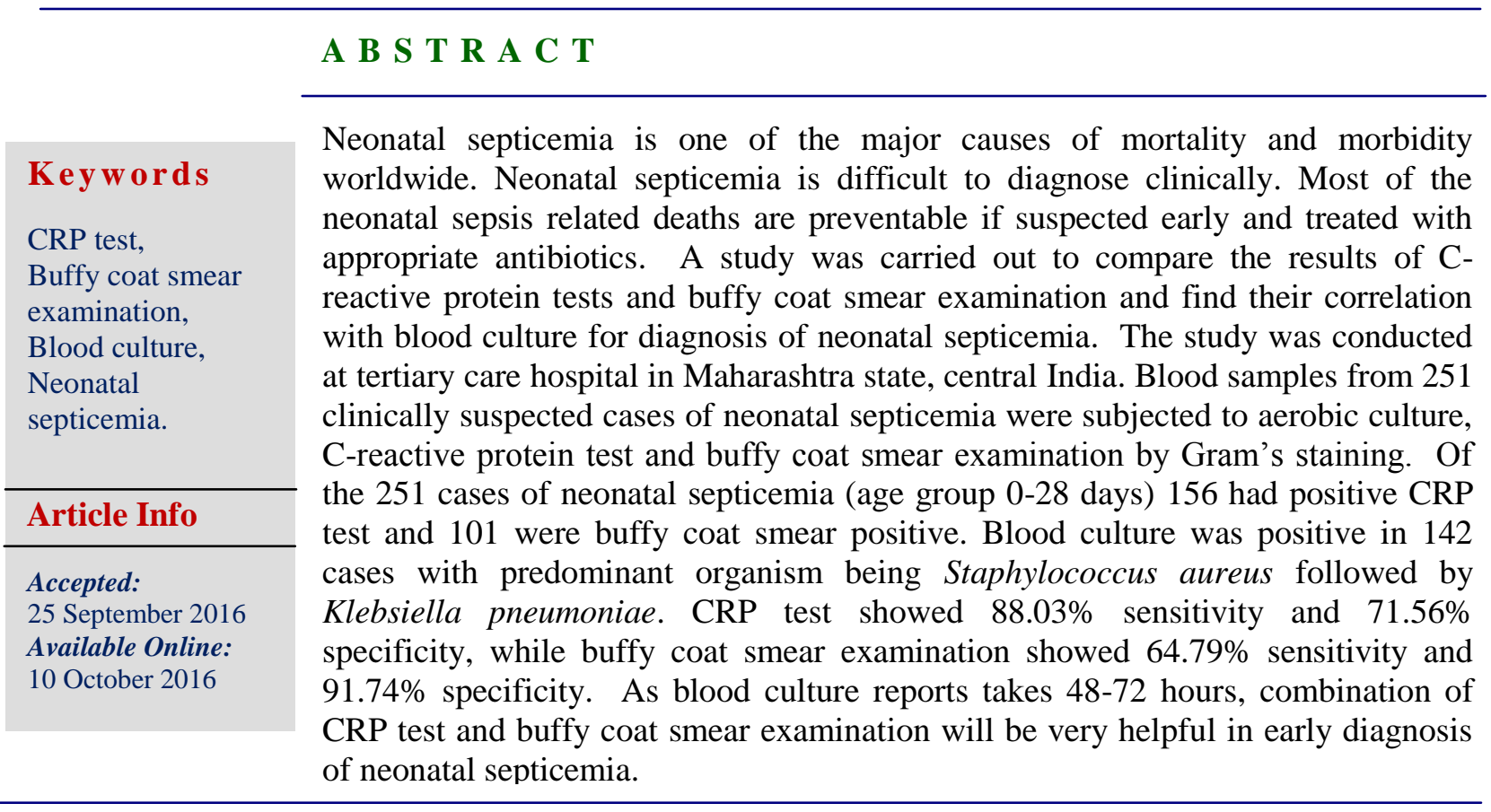

\section{Introduction}

Neonatal sepsis is a clinical syndrome characterized by signs and symptoms suggestive of infection with or without accompanying bacteremia in first month of life. It encompasses various systemic infections of the newborn such as septicemia, meningitis, pneumonia, arthritis, osteomyelitis and urinary tract infection (Sankar et al., 2008). In spite of great advances in antimicrobial therapy, septicemia continues to be a major cause of mortality and morbidity amongst neonates worldwide (Schaffner et al., 2009 and Kaistha et al., 2009). Bacterial infections are the commonest cause of morbidity and mortality during the neonatal period. World Health Organization has estimated that 1.6 million deaths occurs globally every year due to neonatal infections and $40 \%$ of all neonatal deaths occur in developing counties (Sundaram et al., 2009). Most of the neonatal sepsis related deaths are 
preventable if suspected early and treated with appropriate antibiotics (Zakaria et al., 2011). Neonatal septicemia is difficult to diagnose clinically as it presents with non specific signs and symptoms. Traditional method like blood culture is considered as gold standard for diagnosis of septicemia. It takes at least 48-72 hrs to confirm diagnosis and it causes delay in initiation of appropriate therapy. It is also possible that a pseudo-negative result may be obtained in some cases (Panero et al., 1997). Hence certain rapid diagnostic tests were developed to diagnose septicemia and initiation of appropriate therapy to prevent morbidity and mortality.

In the present study rapid diagnostic tests like C-reactive protein tests and buffy coat smear examination by Gram's stain were evaluated in clinically suspected cases of neonatal septicemia in correlation with blood culture.

\section{Materials and Methods}

The present observational study was conducted in the Department of Microbiology at tertiary care hospital in Maharashtra state, central India from January 2014 to June 2015, after obtaining the necessary permission from Institutional Ethical Committee (IEC).

251 blood samples from clinically suspected cases of neonatal septicemia were included in the study. All newborn babies aged 0-28 days presenting with one or more clinical features suggestive of septicemia like poor feeding, lethargy, hypothermia, hyperthermia, jaundice apnea, tachypnea, tachycardia, abdominal distention and vomiting, diarrhoea, skin mottling, bleeding tendencies, seizures and having one or more risk factors like low birth weight, prematurity, birth asphyxia, Premature rupture of membranes, prolonged labour, instrumentation, home delivery, were included in the present study. Neonates with clinical features suggestive of septicemia receiving antibiotics before collection of blood sample and with gross congenital malformations were excluded from the study. Blood samples were processed for Creactive protein test, buffy coat smear examination and blood culture.

\section{C-Reactive Protein Test (CRP)}

This test was done by using diagnostic kit for in-vitro detection of CRP in human serum by rapid slide latex agglutination method using kit commercially supplied by Beacon Diagnostics Pvt. Ltd.

\section{Qualitative slide test}

$40 \mathrm{ul}$ of serum sample, one drop of positive serum control and one drop of negative serum control were pipetted into separate wells of the slide. One drop each of CRP latex reagent was added to all samples, mixed with separate sticks, rotated for 2 minutes and results were read. Presence of visible agglutination was taken as positive test result and indicates presence of CRP in the test serum. No agglutination was taken as negative test and indicates absence of CRP in test serum.

\section{Semi quantitative test}

Samples showing positive test by qualitative method were further tested by semi quantitative method to determine the approximate amount of CRP in $\mathrm{mg} / \mathrm{dl}$ present in serum.

For this test serum was diluted using normal saline serially in the ratio of $1: 2,1: 4,1: 8$, 1:16, 1:32 and 1:64 using normal saline. One drop of CRP reagent was added in each 
of these circle and mixed with sticks, rotated for 2 minutes and results were read. The highest dilution of serum showing visible agglutination corresponds to the approximate amount of CRP in $\mathrm{mg} / \mathrm{dl}$ present in the test serum. In the present study a value of CRP $>0.6 \mathrm{mg} / \mathrm{dl}$ was taken as positive test.

\section{Buffy coat smear examination}

Buffy coat smear examination was done by Brook's method (Brooks et al., 1973). One $\mathrm{ml}$ of blood inoculated into a vial containing $10 \mathrm{mg}$ of ethyl diamine tetra acetic acid (EDTA) was used for preparation of buffy coat smear. Using Pasteur's pipette blood was transferred to sterile Wintrobe's tube and centrifuged at $2500 \mathrm{rpm}$ for 15 minutes. The plasma was removed and buffy coat was taken with Pasteur's pipette. Two smears were prepared by using two slide techniques and stained by Gram's staining and examined under oil immersion objective.

\section{Blood culture and antimicrobial sensitivity test}

Blood was collected with aseptic precautions before starting antibiotics and 1 $\mathrm{ml}$ of venous blood was added to each of the two bottles containing $10 \mathrm{ml}$ of sterile glucose broth and bile broth thus making a dilution of 1 in 10 to nullify the natural bacteriostatic/bacteriocidal activity of blood. Both these bottles were incubated under aerobic conditions in the incubator for 7 days.

The first subculture was done after 18-24 hours of incubation, the second on third day and final subculture was done on seventh day. Subcultures were done on nutrient agar, $5 \%$ blood agar and MacConkey's agar plates (Hi Media, Mumbai, India).
The inoculated plates were incubated aerobically in the incubator at $35-37^{\circ} \mathrm{C}$ for 18- 24 hours, and the plates were observed for growth. The organisms were identified on the basis of colony characteristics, Gram's staining and biochemical test as per standard bacteriological techniques (Collee et al., 1996). A provisional report was issued after every subculture and if after 7 days, no growth was obtained, the sample was reported as negative. Antimicrobial sensitivity test was done for all the isolates on Muller Hinton agar using commercially available antibiotic discs, by Kirby-Bauer disk diffusion method as per Clinical Laboratory Standards Institute guidelines (CLSI;2014) All the antibiotic disks were obtained from Hi Media, Mumbai, India. The statistical analysis was done using Open Epi, version 2.3 Statistical Package. The results of CRP test and buffy coat smear were compared with the blood culture results, considering results of blood culture as a gold standard.

\section{Results and Discussion}

Out of 251 cases, 142 cases were blood culture positive thus prevalence of neonatal septicemia in the present study was $56.57 \%$. Out of 142 culture positive cases, 86 $(60.56 \%)$ were Gram positive isolates, and $56(39.44 \%)$ were Gram negative isolates. Staphylococcus aureus (59.15\%) was found to be the most common causative agent followed by Klebsiella pneumoniae $(30.28 \%)$. Other isolated causative agents were, Pseudomonas aeruginosa (4.93\%) Acinetobacter baumannii (2.82\%), Staphylococcus epidermidis (1.41\%), Klebsiella oxytoca $(0.70 \%)$ and Proteus mirabilis $(0.70 \%)$.

As shown in table 1 out of 251 cases, blood culture was positive in $142(56.57 \%)$ cases and negative in 109 (43.43\%) cases, whereas 
CRP was positive in $156(62.15 \%)$ cases and negative in $95(37.85 \%)$ cases. Both blood culture and CRP were positive in 125 $(49.80 \%)$ cases and negative in $78(31.08 \%)$ cases. $17(6.77 \%)$ cases were blood culture positive and CRP negative, while 31 $(12.35 \%)$ cases were blood culture negative and CRP positive. Thus, Sensitivity, Specificity, Positive predictive value and Negative predictive value of CRP were $88.03 \%, 71.56 \%, 80.13 \%$ and $82.11 \%$ respectively.

As shown in table 2 Both blood culture and buffy coat smear were positive in 92 $(36.65 \%)$ cases and negative in 100 (39.84\%) cases. In 50 (19.92\%) cases blood culture was positive where as buffy coat smear was negative and in 09 (3.59\%) cases, blood culture was negative while buffy coat smear was positive. Thus, Sensitivity, Specificity, Positive predictive value and Negative predictive value of buffy coat smear were $64.79 \%, 91.74 \%, 91.09 \%$ and $66.67 \%$ respectively.

Out of 86 Gram positive isolates CRP was positive in $74(59.2 \%)$ cases and false negative in 12 cases, buffy coat smear was positive in $56(60.87 \%)$ cases and false negative in 30 cases. In case of 56 Gram negative isolates CRP was positive in 51 $(40.08 \%)$ cases and false negative in 05 cases, buffy coat smear was positive in 36 $(39.13 \%)$ cases and found to be false negative in 20 cases. Buffy coat smear gave false negative results in 30 out of 86 Gram positive isolates and 20 out of 56 due to Gram negative isolates. In this study buffy coat smear gave false positive in 9 cases, 6 showed Gram positive and 3 showed Gram negative bacteria.

Neonatal septicemia with its high mortality rate still remains a diagnostic and treatment challenge for the neonatal health care providers. An early diagnosis of neonatal septicemia helps the clinician in instituting antibiotic therapy at the earliest, thereby reducing the mortality rates in the neonates. There is no single reliable test for the early definite diagnosis of neonatal septicemia, and therefore, there is a continuing search for a new infection marker. The $\mathrm{C}$-reactive protein has been the most analyzed parameter for the detection of bacterial infections for years and buffy coat smear examination is considered as one of the rapid test available for diagnosis of neonatal septicemia.

In the present study an attempt has been made to find out the efficacy of rapid diagnostic test like buffy coat smear examination and $\mathrm{C}$-reactive protein test in the diagnosis of neonatal septicemia and correlation of these tests with positive blood culture, considering blood culture as a gold standard for diagnosis of neonatal septicemia.

Out of 251 cases, 142 cases were blood culture positive thus prevalence of neonatal septicemia in the present study was $56.57 \%$. Culture positivity rate is highly variable from place to place and positive blood cultures reportedly range from $8 \%$ to $73 \%$ (Wattal et al., 2011) In the present study out of 142 cultures positive cases, $86(60.56 \%)$ were gram positive isolates and $56(39.44 \%)$ were gram negative isolates. Staphylococcus aureus has been found to predominant pathogen $(59.15 \%)$ followed by Klebsiella pneumoniae (30.28\%), Pseudomonas aeruginosa (4.93\%) Acinetobacter baumannii (2.82\%), Staphylococcus epidermidis (1.41\%), Klebsiella oxytoca $(0.70 \%)$ and Proteus mirabilis $(0.70 \%)$. This predominance of Staphylococcus aureus was correlated with the study done by Karthikeyan et al., (2001) and Shrinivasa et al., (2014). 
CRP is an acute-phase reactant which is synthesized by the liver, diagnose and follow the course of infection in neonate (Kawamura et al., 1995). Its advantages include its very low serum levels in normal infants, a rapid rise within 12 to 24 hours of sepsis and a large incremental increase thereafter.

In the present study, out of 251 cases, CRP was positive in 156 cases. Considering blood culture as a gold standard test for diagnosis of neonatal septicemia, out of 142 culture positive cases, 125 cases were true positive, 17 cases were false negative and 31 cases were false positive. Thus sensitivity of CRP test was $88.03 \%$, specificity was $71.56 \%$, positive predictive value was $80.13 \%$ and negative predictive value was 82.11\%. De et al., (1998) reported sensitivity and specificity, positive predictive value and negative predictive value of CRP as $100 \%, 87.30 \%, 88.30 \%$ and 100\%. Prasad et al., (2012) reported sensitivity and specificity, positive predictive value and negative predictive value of CRP as $87.83 \%, 71.43 \%, 73.45 \%$, $86.21 \%$ respectively. Gandhi et al., (2012) reported sensitivity and specificity of CRP as 100\%, 75\% and Yadav et al., (2015) reported sensitivity and specificity of CRP as $88 \%$, and $84 \%$ respectively. Thus from the present study and above referred studies it is seen that sensitivity of CRP for diagnosis of neonatal septicemia varies from, $87.83 \%$ to $100 \%$. As estimation of other inflammatory markers is costly therefore they cannot be used in clinical and routine application in low resource settings. Therefore, CRP being easily measurable and more affordable can be conveniently used as a good marker for the diagnosis of neonatal sepsis especially in developing countries with limited resources.

Buffy coat smear examination is easy, cheap and reproducible technique of demonstrating micro-organisms. Out of 251 cases, buffy coat was positive in 101 cases and out of 142 culture positive cases, 92 were true positive and 50 were false negative and 9 were false positive. Thus sensitivity of buffy coat smear, specificity, positive predictive value and negative predictive value was $64.79 \%, 91.74 \%, 91.09 \%$ and $66.67 \%$ respectively.

Table.1 Comparison of results of blood culture and CRP test

\begin{tabular}{|c|c|c|c|}
\hline & $\begin{array}{c}\text { Blood culture } \\
\text { positive }\end{array}$ & $\begin{array}{c}\text { Blood culture } \\
\text { negative }\end{array}$ & Total \\
\hline CRP positive & 125 & 31 & 156 \\
\hline CRP negative & 17 & 78 & 95 \\
\hline Total & 142 & 109 & 251 \\
\hline
\end{tabular}

Table.2 Comparison of results of blood culture and buffy coat smear

\begin{tabular}{|c|c|c|c|}
\hline & $\begin{array}{c}\text { Blood culture } \\
\text { positive }\end{array}$ & $\begin{array}{c}\text { Blood culture } \\
\text { negative }\end{array}$ & Total \\
\hline BCS positive & 92 & 09 & 101 \\
\hline BCS negative & 50 & 100 & 150 \\
\hline Total & 142 & 109 & 251 \\
\hline
\end{tabular}


Table.3 Correlation among positive blood culture isolates, positive CRP test and positive buffy coat smear

\begin{tabular}{|c|c|c|c|c|}
\hline $\begin{array}{l}\text { Sr. } \\
\text { No. }\end{array}$ & Bacterial isolates & $\begin{array}{c}\text { Blood culture } \\
\text { positive }\end{array}$ & $\begin{array}{l}\text { CRP test } \\
\text { positive }\end{array}$ & $\begin{array}{c}\text { Buffy coat } \\
\text { smear positive }\end{array}$ \\
\hline \multicolumn{5}{|c|}{ Gram Positive $(n=86)$} \\
\hline 1. & $\begin{array}{l}\text { Staphylococcus } \\
\text { aureus }\end{array}$ & 84 & 72 & 55 \\
\hline 2. & $\begin{array}{l}\text { Staphylococcus } \\
\text { epidermidis }\end{array}$ & 02 & 02 & 01 \\
\hline \multicolumn{5}{|c|}{ Gram negative $(n=56)$} \\
\hline 3. & $\begin{array}{l}\text { Klebsiella } \\
\text { pneumoniae }\end{array}$ & 43 & 39 & 29 \\
\hline 4. & Klebsiella oxytoca & 01 & 01 & 00 \\
\hline 5. & $\begin{array}{l}\text { Pseudomonas } \\
\text { aeruginosa }\end{array}$ & 07 & 07 & 04 \\
\hline 6. & Proteus mirabilis & 01 & 01 & 00 \\
\hline 7. & $\begin{array}{l}\text { Acinetobacter } \\
\text { baumannii }\end{array}$ & 04 & 03 & 03 \\
\hline & Total & 142 & 125 & 92 \\
\hline
\end{tabular}

\section{Graph.1}

Graph 1: Distribution of bacterial isolates causing septicemia

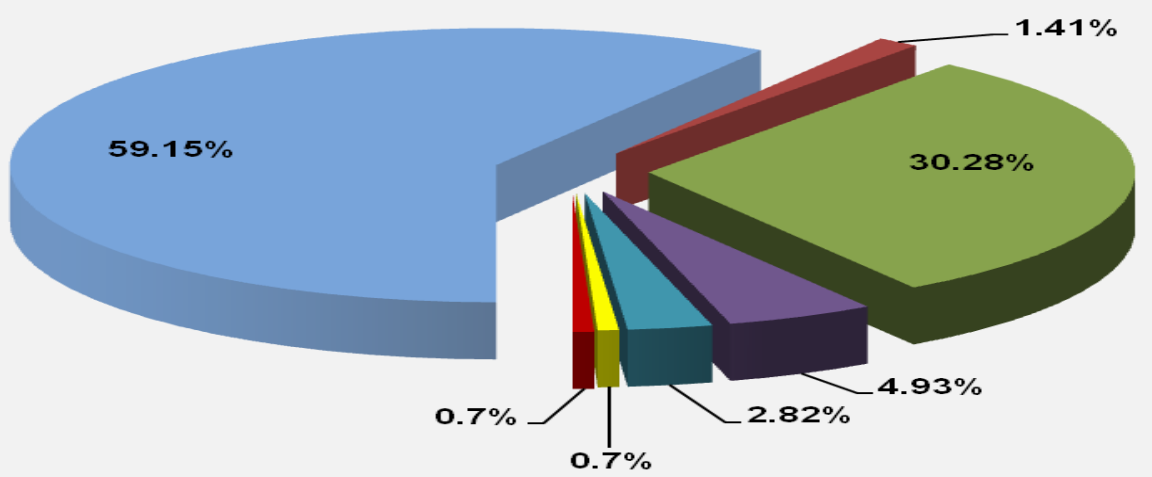

Staphylococcus aureus

- Klebsiella pneumoniae

Acinetobacter baumannii

- Klebsiella oxytoca

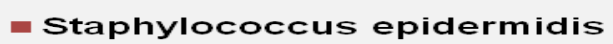

- Pseudomonas aeruginosa

Proteus mirabilis 
Although buffy coat smear examination is considered as one of the rapid test available for diagnosis of neonatal septicemia, variable results were reported by various authors, De et al., (1998) reported sensitivity and specificity, positive predictive value and negative predictive value of CRP as 76.50 $\%, \quad 91.74 \%, \quad 89.30 \%$ and $80.20 \%$ respectively, Jha et al., (2011) reported sensitivity and specificity, positive predictive value and negative predictive value of CRP as $84.34 \%, 91.04 \%, 92.11 \%$ and $82.43 \%$ respectively.

The specificity and positive predictive value of buffy coat smear reported by De et al., (1998) and Jha et al., (2011) are comparable with the results of the present study. For rapid diagnosis of neonatal septicemia the combination of buffy coat smear examination and C-reactive protein test will be very useful. Both of these tests are rapid and easy to perform, buffy coat smear is cheap and easily reproducible and CRP test is highly sensitive in cases of neonatal septicemia.

Although blood culture is gold standard for the diagnosis of neonatal septicemia, it takes at least 48 to 72 hours to obtain the reports of diagnosis which causes delay in initiation of appropriate therapy. Therefore, rapid diagnostic tests like buffy coat smear examination and C-reactive protein test will be helpful in early diagnosis of neonatal septicemia to reduce morbidity and mortality in neonates.

\section{References}

Brooks G.F., Pribble A.H., Beaty H.N. 1973. Early Diagnosis of Bacteremia by Buffy Coat Examinations. Arch Intern Med., 132: 673-675.

CLSI. 2014. Performance Standards for Antimicrobial Susceptibility Testing.
Twenty-Fourth Informational

Supplement. CLSI document M100-

S24. Wayne, PA: Clinical and Laboratory Standards Institute.

Collee, J.G., Fraser, A.G., Marmion, B.P., Simmons, A. Mackie and Mc Cartney. 1996. Practical Medical Microbiology. $14^{\text {th }}$ ed. Edinburgh: Churchill Livingstone, 113-149.

De, A., Saraswathi, K., Gogate, A., Raghavan, K. 1998. C-Reactive Protein and Buffy Coat Smear in Early Diagnosis of Childhood Septicemia. Indian J. Pathol. Microbiol., 41: 2326.

Gandhi, T.N., Patel, M.G., Jain, M.R., Saxena, R.B., Bhuva, P.J. 2012. Utility of C-reactive protein as inflammatory marker in early diagnosis of neonatal Septicaemia: a cross sectional study. Nat. J. Med. Res., 2: 481-483.

Jha, B.K., Singh, Y.I., Mahadevmurthy, S., Chaudhary, N.K. 2011. Septicemia detection by blood buffy coat smear in primary health care centers. Nepal Med. Coll. J., 7: 19-23.

Kaistha, N., Mehta, M., Singla, N., Garg, R., Chander, J. 2009. Neonatal septicemia isolates and resistance patterns in a tertiary care hospital of North India. $J$. Infect. Dev. Ctries, 4: 55-57.

Karthikeyan, G., Premkumar, K. 2001. Neonatal Sepsis: Staphylococcus aureus as the Predominant Pathogen. Indian J. Pediatr., 68: 715-717.

Kawamura, M. and Nishida, H. 1995. The usefulness of serial C-reactive protein measurement In managing neonatal infection. Acta Paediatr., 84: 10-13.

Panero, A., Pacifico, L., Rossi, N., Mancuso, G., Stegagno, M., Chiesa, C. 1997. Interleukin 6 in neonates with early and late onset infections. Paediatr. Infect. Dis. J., 16: 370-375.

Prasad, R., Basavraj, K.N., Antony, B. 2012. 
Rapid diagnosis of neonatal septicemia by buffy coat smear examination and $\mathrm{C}$-reactive protein test in correlation with blood culture. Int. J. Biol. Med. Res., 3: 1658-1661.

Sankar, M.J., Agarwal, R., Deorari, A.K., Paul, V.K. 2008. Sepsis in the newborn. Indian J. Pediatr., 75: 261266.

Schaffner, J., Chochua, S., Kourbatova, E.V., et al. 2009. High mortality among patients with positive blood cultures at a children's hospital in Tbilisi, Georgia. J. Infect. Dev. Ctries, 3: 267-272.

Srinivasa, S. and Arunkumar, D. 2014. Bacterial isolates and their susceptibility patterns in neonatal sepsis. Curr. Pediatr. Res., 18: 83-86.
Sundaram, V., Kumar. P., Datta, S., et al. 2009. Blood culture confirmed bacterial sepsis in neonates in a North Indian tertiary care center: Changes over the last decade. Jpn. J. Infect. Dis., 62: 46-50.

Wattal, C. and Oberoi, J.K. 2011. Neonatal sepsis. Indian J. Pediatr., 78: 473-474.

Yadav, M., Lekharu, R., Darjis. 2015. Role of C-reactive protein in early diagnosis of neonatal sepsis. Int. J. Curr. Res., 7: 18280-18282.

Zakaria, B.P., Bhat, V., Harish, B.N., Babu, T.A., Joseph, N.M. 2011. Neonatal sepsis in a tertiary care hospital in South India: Bacteriological profile and antibiotic sensitivity pattern. Indian J. Pediatr., 78: 413-417.

\section{How to cite this article:}

Archana P. Nikam and Pramod R. Bhise. 2016. C - Reactive Protein Test and Buffy Coat Smear Examination in Early Diagnosis of Neonatal Septicemia. Int.J.Curr.Microbiol.App.Sci. 5(10): 867-874. doi: http://dx.doi.org/10.20546/ijcmas.2016.510.095 